\title{
Design of High Speed Full Adder using Improved Differential Split Logic Technique for 130nm Technology and its Implementation in making ALU
}

\author{
Gurleen Kaur \\ Mtech Microelectronics \\ UIET, Panjab University \\ Chandigarh, UT India
}

\author{
Arvind Kumar \\ Assistant Professor, ECE \\ UIET, Panjab University \\ Chandigarh, UT India
}

\author{
Jatinder Singh \\ Assistant Professor, ECE \\ UIET,Panjab University \\ Chandigarh,UT India
}

\begin{abstract}
Adders are the main components in digital designs which are used not only for addition but can be used for multiplication and division too. Adders find use in very large scale integrated circuits from processors (like in arithmetic logic circuits) to application specific integrated circuits. At the same time, high speed computation has become the important part of any digital applications today though low power is a key factor too. In this paper, a high speed full adder using improved differential split logic (DSL) technique is used. We further implement it in 1bit arithmetic logic circuit (ALU). Measurements show that proposed full adder is better than DSL full adder in terms of speed, and further implementation of it in ALU shows that it is better than CMOS ALU in terms of speed, power and power delay product (PDP).
\end{abstract}

\section{General Terms}

Full adder, Differential split level logic (DSL), Differential cascade voltage logic (DCVS), Arithmetic logic circuit (ALU), Power delay product (PDP), Full adder (FA), Multiplexer (Mux).

\section{Keywords}

Differential Split logic, Full adder, Arithmetic logic circuits

\section{INTRODUCTION}

Full adders are the heart of all arithmetic calculations [13]. It is a combinational logic unit that performs all the calculations like addition, subtraction, increment and decrement. There are certain factors that can simply slow down the development of small complex IC chips. These factors are design cost, design productivity and IC fabrication technology. The increasing demand for high speed very large scale integration can be obtained at design levels such as architectural, circuit and layout level .For the circuit design, at this level a proper choice of logic design style for high speed combinational logic circuits should be done. It is because all the important parameters affecting speed are switching capacitance, transition activity and short circuit currents are actually influenced by the chosen logic style. Earlier the parameters like power dissipation, small area and cost factor were given more weight age, but now days speed considerations are also the important factors for the scientific community related to VLSI designs.

In CMOS technology, even if we design high speed full adder, power dissipation is also taken care of. Power dissipation is one of the critical factors which is of two types and is classified into dynamic power and static power. Dynamic power dissipation comes into picture when the circuit is operational and static power dissipation is considered when the circuit is inactive.

Dynamic power is further classified into switching power $\left(P_{\text {switch }}\right.$ and short circuit power $\left(P_{s c}\right)$.Static power is because of leakage power dissipation [7].

There are three major sources of power dissipation which are given in the following equation.

$$
P_{\text {total }}=\alpha \cdot C_{l} \cdot V_{d d}{ }^{2} \cdot f_{c l k}+I_{s c} \cdot V_{d d}+I_{\text {leakage }} \cdot V_{d d}
$$

The first term shows the switching component of power where $\alpha$ is the switching factor, $C_{l}$ is the loading capacitance, $f_{c l k}$ is the clock frequency. The second term represents the dissipation due to short circuit currents. The last term is the dissipation due to leakage currents ( $\left.I_{\text {leakage }}\right)[1]$.

The switching power dissipation in CMOS digital integrated circuits is an important function of the power supply voltage $V_{d d}$ Reduction of power supply voltage is an important way to limit the power dissipation. But then limiting the power dissipation, results in increased circuit delay. The given equation tells about the dependence of delay on power supply voltage:

$$
T_{d} \propto C_{l} V_{d d} k\left(V_{d d}-V_{t h}\right)^{\alpha}
$$

Where $\mathrm{k}=$ transistor aspect ratio (W/L), $V_{t h}=$ transistor threshold voltage, $\alpha=$ velocity saturation index which varies between 1 and 2 .

On decreasing the power supply voltage, power dissipation though is reduced, but instead the delay increases as the threshold voltage reaches near the supply voltage. This problem is overcome by using the low power differential split logic technique.

Section II gives a brief introduction of conventional full adder and the various logic styles that can be used to make full adders. Section III describes about the propagation delay analysis in DCVS and DSL circuits.

Section IV describes about the proposed DSL full adder and its implementation using the Tanner v 13.0 tool.

Section V further discusses about the implementation of these adders in designing arithmetic logic circuit.

Section VI tells about the conclusion. 


\section{LOGIC DESIGN STYLES}

\subsection{Conventional CMOS}

The schematic diagram of a conventional CMOS adder is shown in the given figure:

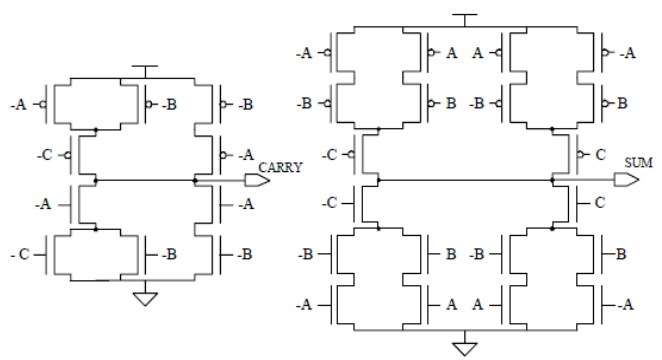

Fig 1: CMOS full adder [2]

Signals noted with '-'are basically complementary signals. The width of the transistors is increased to obtain a reasonable conducting current to drive the capacitive loads which further results in increased capacitance and high propagation delay.

\subsection{Complementary pass transistor logic}

The schematic diagram of CPL adder is shown in the diagram.

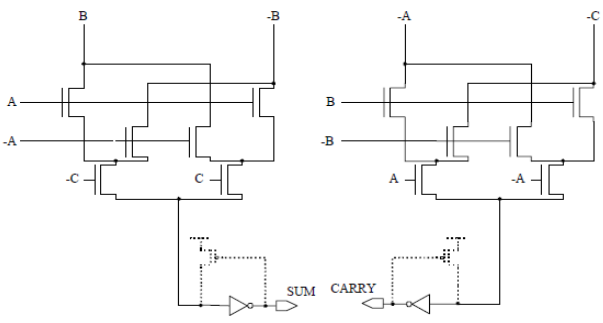

Fig 2: CPL full adder [2]

In CPL, the logic functions are implemented by the use of only an NMOS. This basically results in low input capacitance and high speed operation.CPL circuits consume less power than conventional static logic circuits because the logic output voltage swing of the pass transistor is less than the supply voltage level.

\subsection{Differential cascode voltage switch logic (DCVSL)}

DCVS have reduced transistor counts over NAND/NOR implementations. DCVS has got better performance of about 4times as compared to the CMOS/NMOS NAND/NOR circuits and maintaining the low power circuitry. The schematic diagram of the full adder is shown

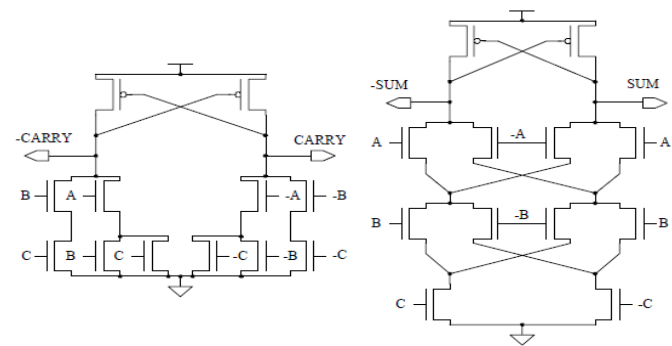

Fig 3: DCVS full adder [2]
Two complementary NMOS transistors set of switching trees are designed to connect to a couple of cross coupled PMOS transistors. One of the outputs is pulled down by NMOS network depending on the differential inputs. The input capacitance is two or three times smaller than that of conventional CMOS adder because the inputs drive only the NMOS transistors.

\subsection{Differential split level logic (DSL)}

Two NMOS transistors with their gates connected to the reference voltage are added to reduce the logic swing at the output nodes.

$$
V_{\text {ref }}=\left(V_{d d} / 2\right)+V_{t h}
$$

Where $V_{t h}=$ NMOS threshold voltage.

So the output nodes are clamped at the half power supply voltage, hence the operation of the circuit becomes faster than the DCVSL operation. But these circuits dissipate high static power dissipation due to the incomplete turn off of the cross coupled circuits. Addition of two more NMOS transistors results in the increase of area. The circuit diagram of DSL full adder is shown.

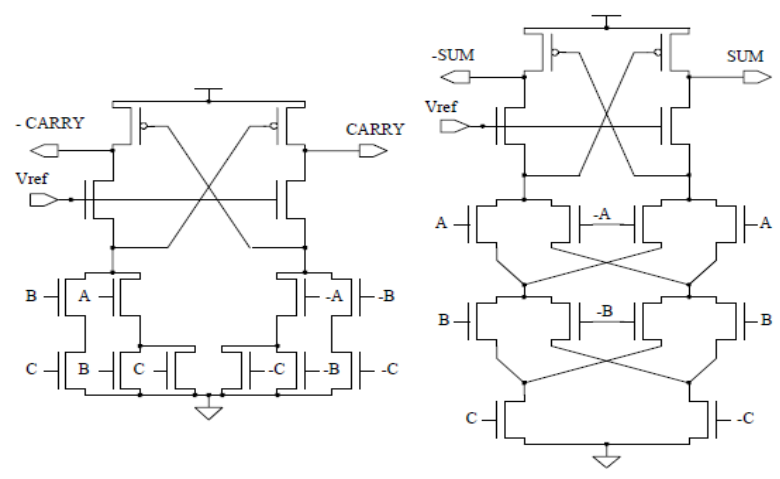

Fig 4: DSL full adder [2]

\section{DELAY IN DSL}

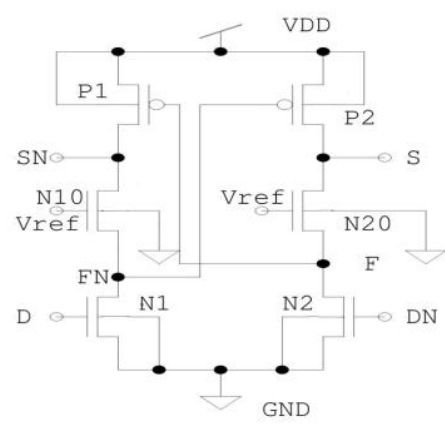

Fig 5: Basic DSL circuit

The propagation delay of a logic gate is defined as the difference between an input and output change [5]. The delay $t_{d}$ is very well shown as the time difference between $V_{I}$ and $\mathrm{V}_{\mathrm{Q}}$, so that it goes through $0.5 \mathrm{~V}_{\mathrm{dd}}$. The input/output waveforms of half DSL logic circuit is shown here in figure 6 [3]. 


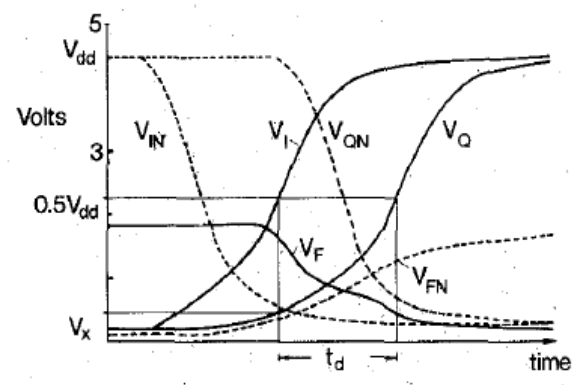

Fig 6: Input /output waveforms of half DSL circuit

The given figure of half DSL logic circuit is considered for the delay analysis.

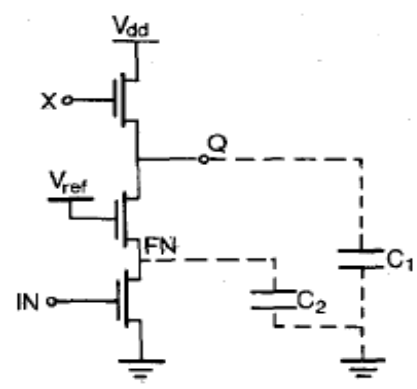

Fig 7: Half DSL circuit

Voltage change at $\mathrm{Q}$ is $\left(0.5 V_{d d^{-}} V_{x}\right)$. Hence, if $\mathrm{C}_{1}$ is the capacitance at $\mathrm{Q}$ and $I_{C l}$, the current which charges it during the propagation delay then $t_{d}$ can be expressed as:

$$
T_{d}=C_{l}\left(0.5 V_{d d}-V_{x}\right) / I_{c 1}
$$

Moreover $t_{d}$ can be expressed in term of the current of $I_{\text {pmos }}$ which flows through $\mathrm{P}_{2}$ during the propagation delay, which not only charges the capacitance at $\mathrm{C}_{1}$ but also charges the capacitance at node $F_{n}$. Referring to the capacitance $C_{2}$, voltage change across it during the propagation delay $t_{d}$ is $V_{\text {fn. }}$ Considering the $I_{p m o s}, t_{d}$ is expressed as follows :

$$
\begin{gathered}
T_{d}=\left(C_{1}\left(0.5 V_{d d}-V_{x}\right)+C_{2}\left(V_{f n}\right)\right) / I_{p m o s} \\
\text { Where } V_{f u}=K\left(V_{r e f}-V_{m 2}\right) \\
K=A+B\left(C_{2} / C_{1}\right) \\
I_{p m o s}=\left(W_{n} / L_{n}\right) A(\beta / 2)\left(V_{d d}-B\left(V_{r f}-V_{m}\right)-V_{w}\right)^{2}
\end{gathered}
$$

\section{IMPLEMENTATION OF PROPOSED FULL ADDER}

In this modified DSL full adder is proposed. Two resistors are added in series to the reference NMOS N10 and N20 transistors in the DSL circuit in figure 5 which will switch on the PMOS transistors more quickly .This helps in decreasing the delay in the full adder. The worst case delay is considered for the measurements. The transistor $\mathrm{W} / \mathrm{L}$ ratio is 1.66.Tanner v13.0 too is used with the $130 \mathrm{~nm}$ technology file. The schematic diagram of the proposed full adder is shown.

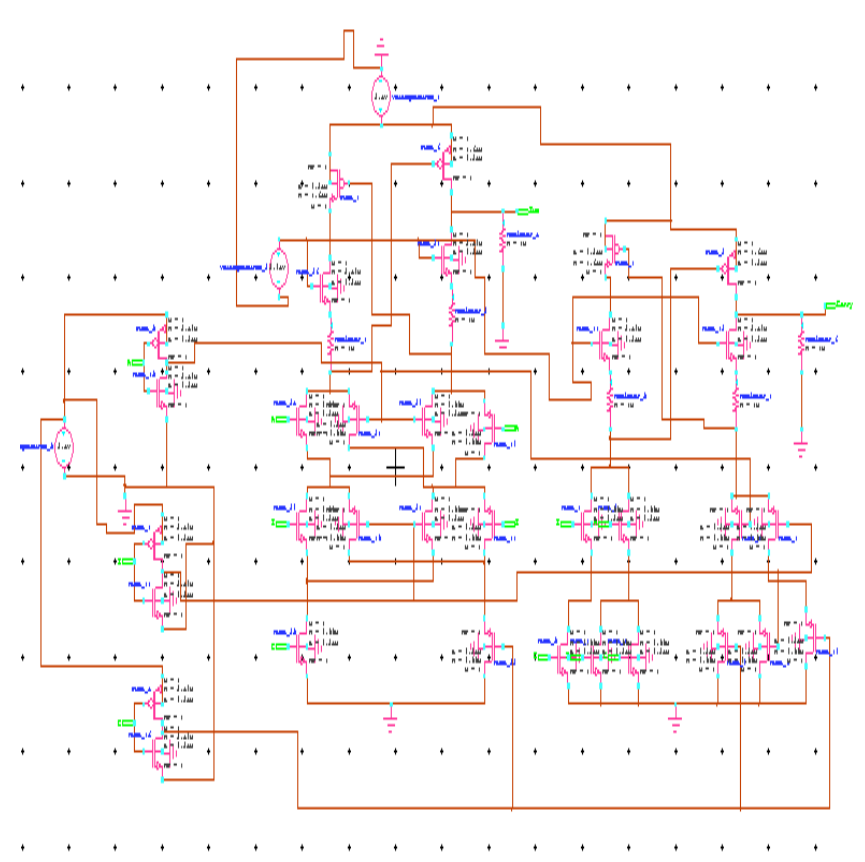

Fig 8: Proposed DSL full adder

The above proposed full adder is further modified by putting capacitor in parallel with the resistors that are added in series to the reference transistors in figure 9 .

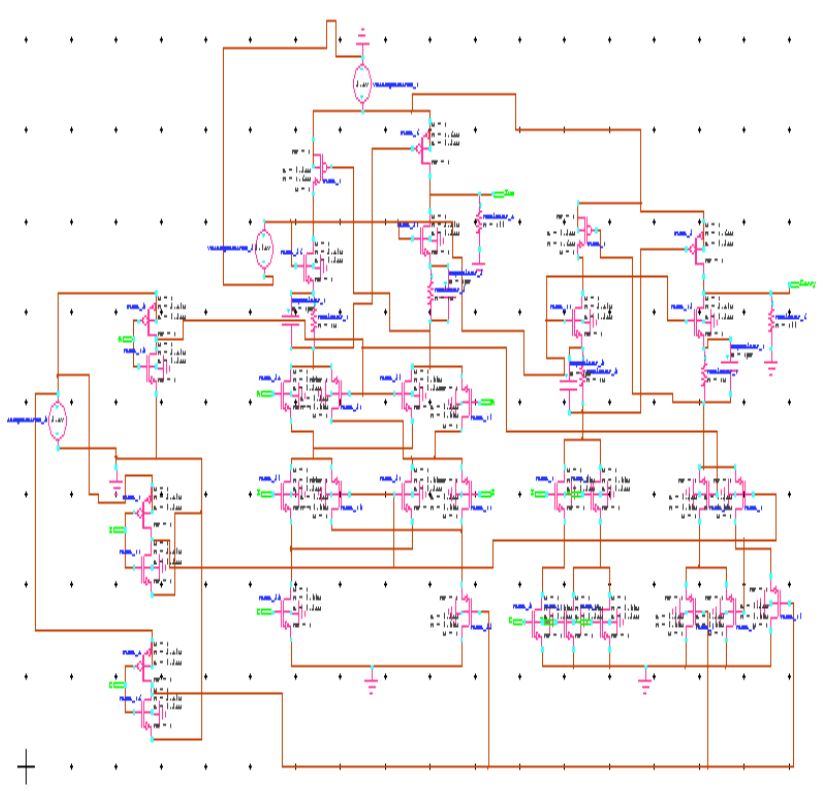

Fig 9: Proposed DSL full adder with capacitance

\subsection{Simulation Results}

The table showing simulation results with proposed full adder starting from resistor $\mathrm{R}=0$ and with increasing resistances is shown. The power voltage source is $2.5 \mathrm{~V}$ and the reference voltage is $2.05 \mathrm{~V}$. 
Table 1.Delay $\left(10^{-10} \mathrm{~ns}\right)$ calculations wrt increasing resistance (ohms)

\begin{tabular}{|c|c|c|c|}
\hline $\begin{array}{c}\text { Resistan } \\
\text { ce }\end{array}$ & $\begin{array}{c}\text { Delay }(C=0 \\
\text { pF })\end{array}$ & $\begin{array}{c}\text { Delay }(C=0.000 \\
1 p F)\end{array}$ & $\begin{array}{c}\operatorname{Delay}(C=0.00 \\
1 p F)\end{array}$ \\
\hline 0 & 1.4846 & 1.4846 & 1.4846 \\
\hline 100 & 1.4577 & 1.4577 & 1.4577 \\
\hline 200 & 1.4341 & 1.4341 & 1.4343 \\
\hline 300 & 1.4220 & 1.4220 & 1.4224 \\
\hline 400 & 1.4104 & 1.4104 & 1.4109 \\
\hline 500 & 1.3977 & 1.3978 & 1.3986 \\
\hline 1000 & 1.3449 & 1.3452 & 1.3481 \\
\hline 1100 & 1.3306 & 1.3309 & 1.3343 \\
\hline 1200 & 1.3224 & 1.3231 & 1.3285 \\
\hline 1300 & 1.3170 & 1.3177 & 1.3181 \\
\hline 1400 & 1.3149 & 1.3108 & 1.3179 \\
\hline 1500 & 1.3156 & 1.3164 & 1.3202 \\
\hline 1600 & 1.3182 & 1.3191 & 1.3241 \\
\hline 1700 & 1.3099 & 1.3111 & 1.3218 \\
\hline 1800 & 1.3034 & 1.3046 & 1.3160 \\
\hline 1900 & 1.2993 & 1.3007 & 1.3127 \\
\hline
\end{tabular}

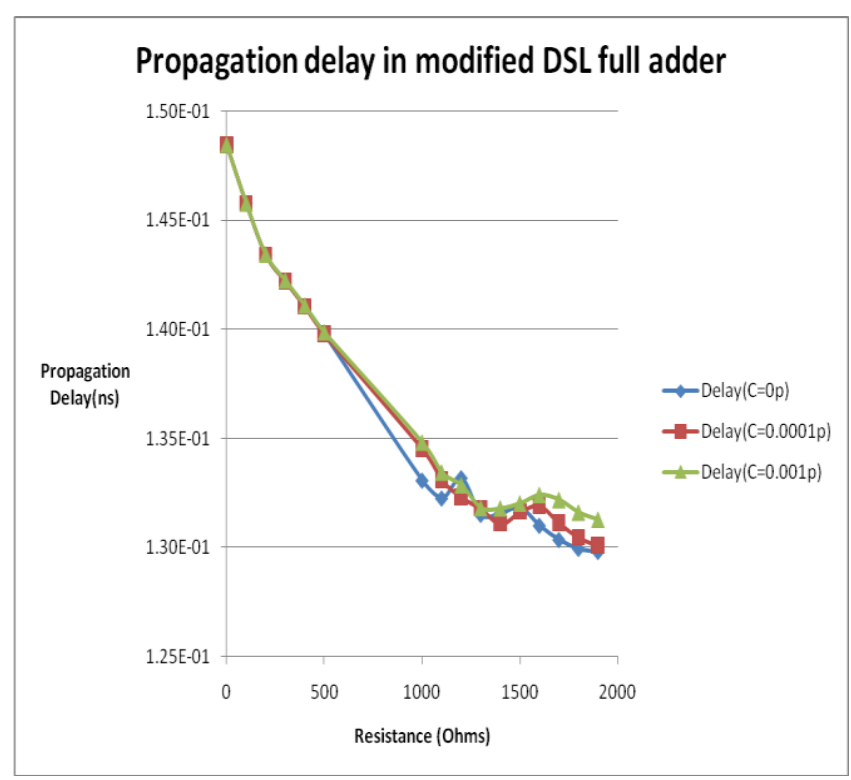

Fig 10: Graph for propagation delay with increasing resistance
Table 2.Power consumption ( $\mu$ w) calculations wrt increasing resistance (ohms)

\begin{tabular}{|c|c|c|c|}
\hline $\begin{array}{c}\text { Resista } \\
\text { nce }\end{array}$ & $\begin{array}{c}\text { Power }(C=0 \\
\text { pF })\end{array}$ & $\begin{array}{c}\text { Power }(C=0.000 \\
1 \mathrm{pF})\end{array}$ & $\begin{array}{c}\text { Power }(\mathrm{C}=0.00 \\
1 \mathrm{pF})\end{array}$ \\
\hline 0 & 7.977327 & 7.977327 & 7.977327 \\
\hline 100 & 8.59742 & 8.597420 & 8.597418 \\
\hline 200 & 9.220806 & 9.220805 & 9.220798 \\
\hline 300 & 9.845852 & 9.84585 & 9.845835 \\
\hline 400 & 10.471105 & 10.471102 & 10.471104 \\
\hline 500 & 11.095287 & 11.095282 & 11.095239 \\
\hline 1000 & 14.16555 & 14.165531 & 14.165364 \\
\hline 1100 & 14.764302 & 14.76428 & 14.764161 \\
\hline 1200 & 15.356704 & 15.356677 & 15.356677 \\
\hline 1300 & 15.942382 & 15.942351 & 15.356442 \\
\hline 1400 & 16.521041 & 16.521006 & 16.520694 \\
\hline 1500 & 17.092466 & 17.092426 & 17.092072 \\
\hline 1600 & 17.656211 & 17.656167 & 17.655773 \\
\hline 1700 & 18.212428 & 18.212379 & 18.211942 \\
\hline 1800 & 18.760887 & 18.760833 & 18.760351 \\
\hline 1900 & 19.575752 & 19.575789 & 19.576158 \\
\hline
\end{tabular}

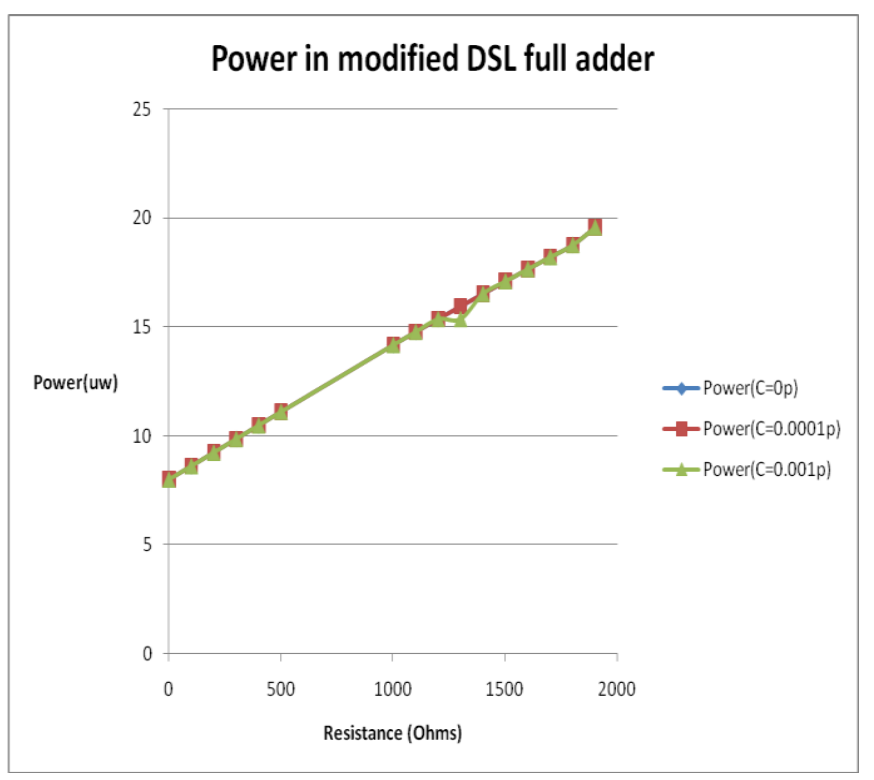

Fig 11: Graph for power consumption with increasing resistance 
Table 3.PDP ( $\mu \mathrm{w}$ ns) calculations wrt increasing resistance (ohms)

\begin{tabular}{|c|c|c|c|}
\hline $\begin{array}{c}\text { Resistan } \\
\text { ce }\end{array}$ & $\begin{array}{c}\operatorname{PDP}(C=0 p \\
F)\end{array}$ & $\begin{array}{c}\mathrm{PDP}(\mathrm{C}=0.0001 \\
\text { pF })\end{array}$ & $\begin{array}{c}\mathrm{PDP}(\mathrm{C}=0.001 \\
\text { pF })\end{array}$ \\
\hline 0 & $\begin{array}{c}1.18431396 \\
6\end{array}$ & 1.184313966 & 1.184313966 \\
\hline 100 & $\begin{array}{c}1.25324591 \\
3\end{array}$ & 1.253245913 & 1.253245622 \\
\hline 200 & $\begin{array}{c}1.32235578 \\
8\end{array}$ & 1.322355645 & 1.322539057 \\
\hline 300 & $\begin{array}{c}1.40008015 \\
14\end{array}$ & 1.40007987 & 1.40047157 \\
\hline 400 & $\begin{array}{c}1.47684464 \\
9\end{array}$ & 1.476844226 & 1.477368063 \\
\hline 500 & $\begin{array}{c}1.55078826 \\
4\end{array}$ & 1.550898518 & 1.551780127 \\
\hline 1000 & 1.90512482 & 1.90554723 & 1.909632721 \\
\hline 1100 & $\begin{array}{c}1.96453802 \\
4\end{array}$ & 1.964978025 & 1.969982002 \\
\hline 1200 & $\begin{array}{c}2.03077053 \\
7\end{array}$ & 2.031841008 & 2.040134539 \\
\hline 1300 & $\begin{array}{c}2.09961170 \\
9\end{array}$ & 2.100723591 & 2.02413262 \\
\hline 1400 & $\begin{array}{c}2.17235168 \\
1\end{array}$ & 2.165573466 & 2.177262262 \\
\hline 1500 & $\begin{array}{c}2.24868482 \\
7\end{array}$ & 2.250046959 & 2.256495345 \\
\hline 1600 & $\begin{array}{c}2.32744173 \\
4\end{array}$ & 2.329024989 & 2.337800903 \\
\hline 1700 & $\begin{array}{c}2.38564594 \\
4\end{array}$ & 2.387825011 & 2.407254494 \\
\hline 1800 & $\begin{array}{c}2.44529401 \\
2\end{array}$ & 2.447538273 & 2.468862192 \\
\hline 1900 & $\begin{array}{c}2.54347745 \\
7\end{array}$ & 2.546222875 & 2.569762261 \\
\hline
\end{tabular}

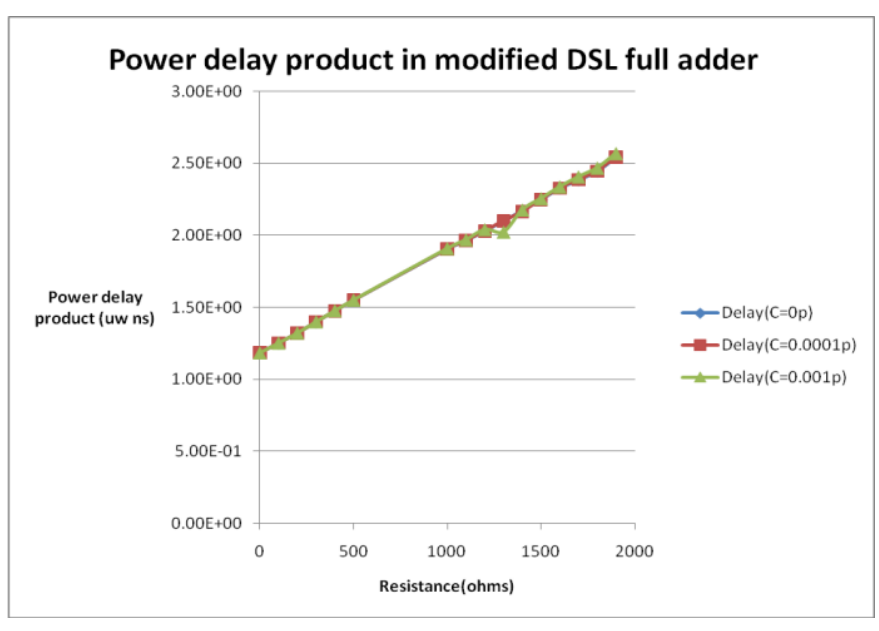

Fig 12: Graph for PDP with increasing resistance

\subsection{Comparison results of proposed FA}

The performance parameters of proposed full adder are compared to the ones given in the paper [10].

Table4. Comparison of performance parameters of FA

\begin{tabular}{|l|l|l|l|}
\hline Logic circuits & \multicolumn{1}{|c|}{ Delay(ns) } & Power( $\boldsymbol{\mu w})$ & PDP( $\boldsymbol{\mu w}$ ns $)$ \\
\hline Proposed FA & 0.12 & 19.57 & 2.569762261 \\
\hline DSL FA & 0.148 & 7.977327 & 1.184313966 \\
\hline CMOS FA & 0.12 & 73.8 & 8.856 \\
\hline CPL FA & 0.11 & 39.1 & 4.301 \\
\hline DCVSL FA & 0.13 & 110.7 & 14.391 \\
\hline P-CPL FA & 0.18 & 21.3 & 3.834 \\
\hline P-DCVSL FA & 0.2 & 103 & 20.06 \\
\hline
\end{tabular}

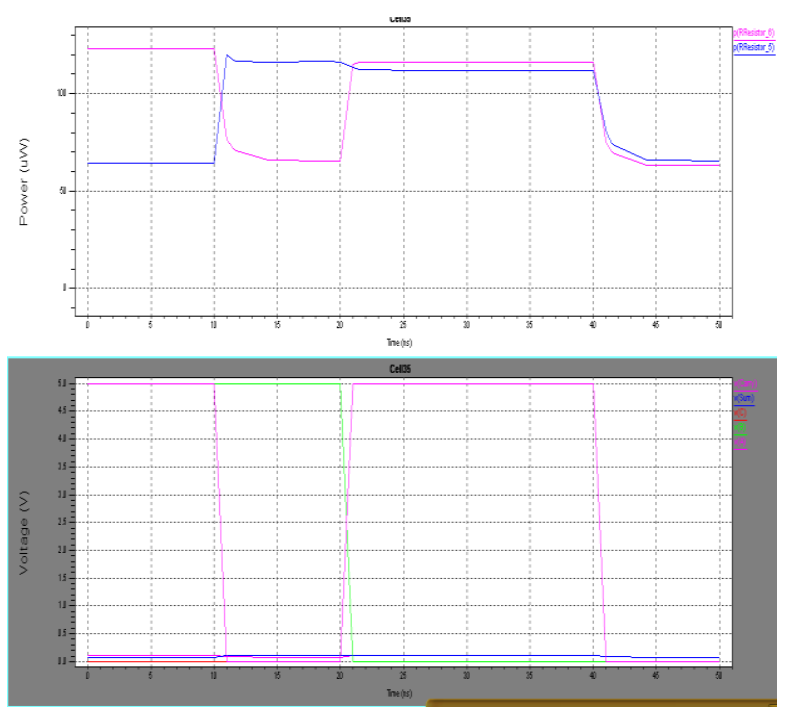

Fig 13: Simulation waveforms of FA (modified) in W-edit 


\section{DESIGN OF ALU USING PROPOSED}

\section{FULL ADDER}

Arithmetic logic unit is designed using the proposed full adder for arithmetic unit. This adder is used for addition, subtraction, increment and decrement by controlling the data inputs to implement these operations [9].Moreover for logic unit AND, OR, XOR and inverter is designed using modified DSL logic which is used in full adders too [11]. For designing ALU, two 4:1 mux and one 2:1 mux are designed using DSL logic [12]. So eight operations are performed by using this ALU shown in table 5.

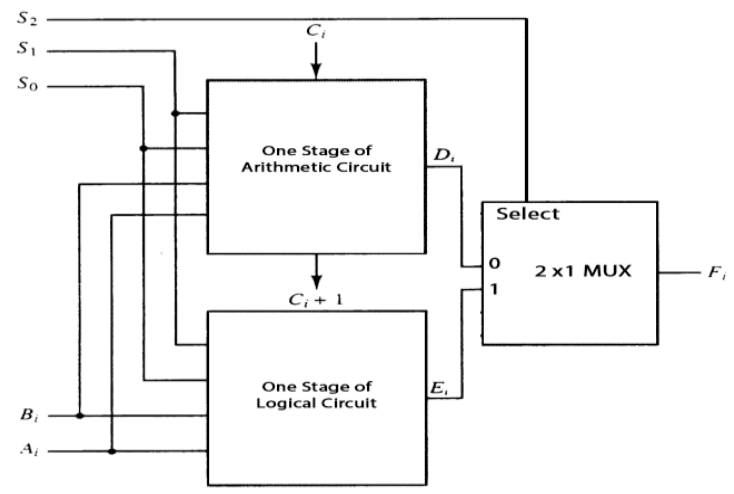

Fig 14: Structure of ALU

Table5. Truth table for ALU

\begin{tabular}{|c|c|c|c|}
\hline S & S1 & S2 & Function \\
\hline 0 & 0 & 0 & Decrement \\
\hline 0 & 0 & 1 & Increment \\
\hline 0 & 1 & 0 & Addition \\
\hline 0 & 1 & 1 & Subtraction \\
\hline 1 & 0 & 0 & AND \\
\hline 1 & 0 & 1 & OR \\
\hline 1 & 1 & 0 & XOR \\
\hline 1 & 1 & 1 & Invert \\
\hline
\end{tabular}

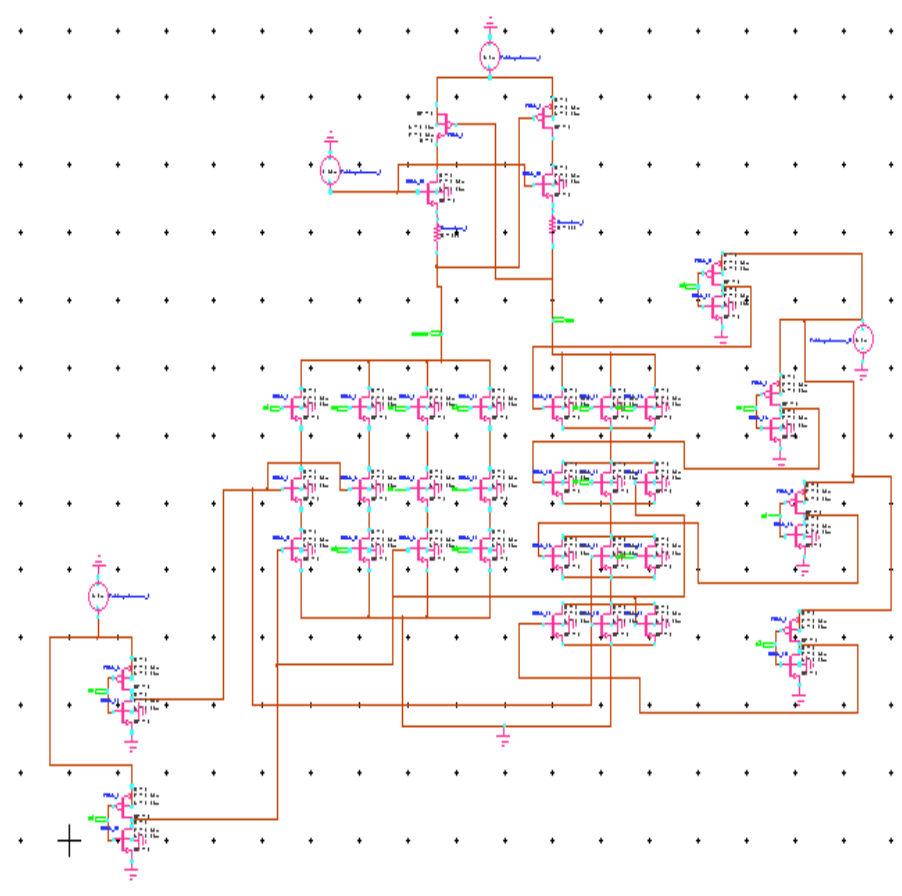

Fig 15: 4:1 Mux circuit schematic

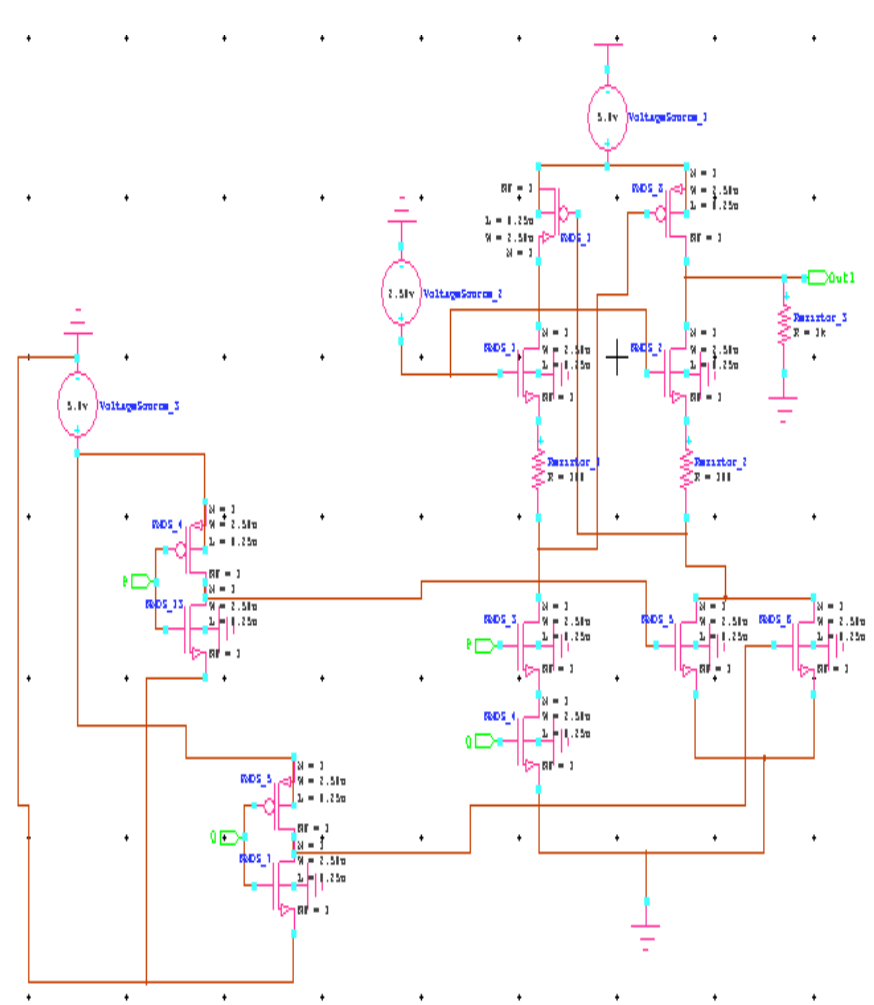

Fig 16: AND circuit schematic 


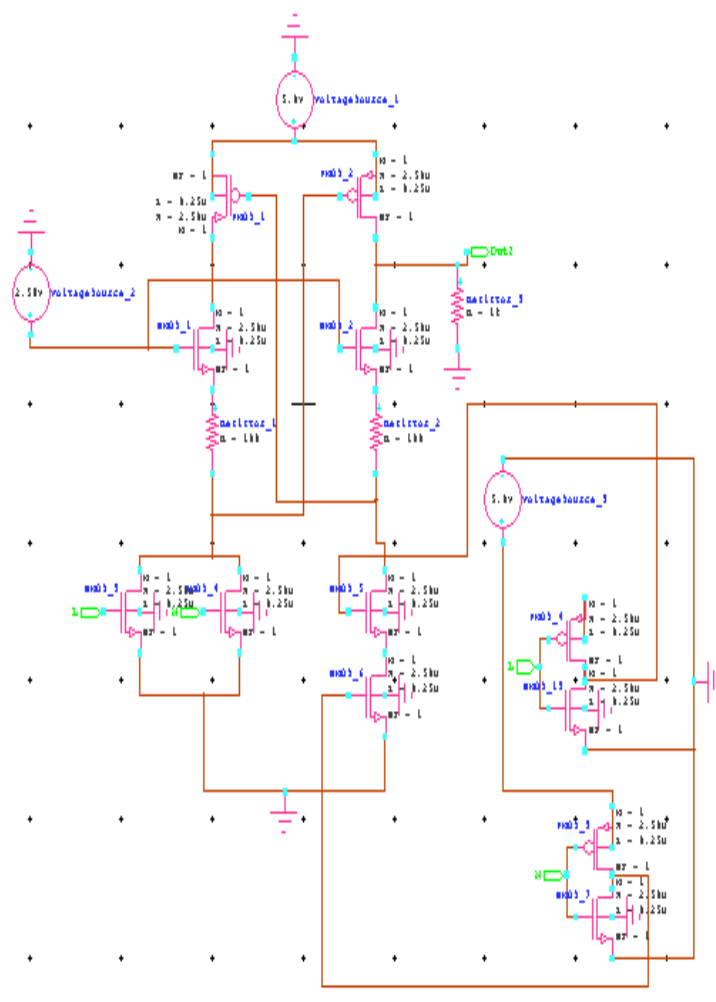

Fig 17: OR circuit schematic

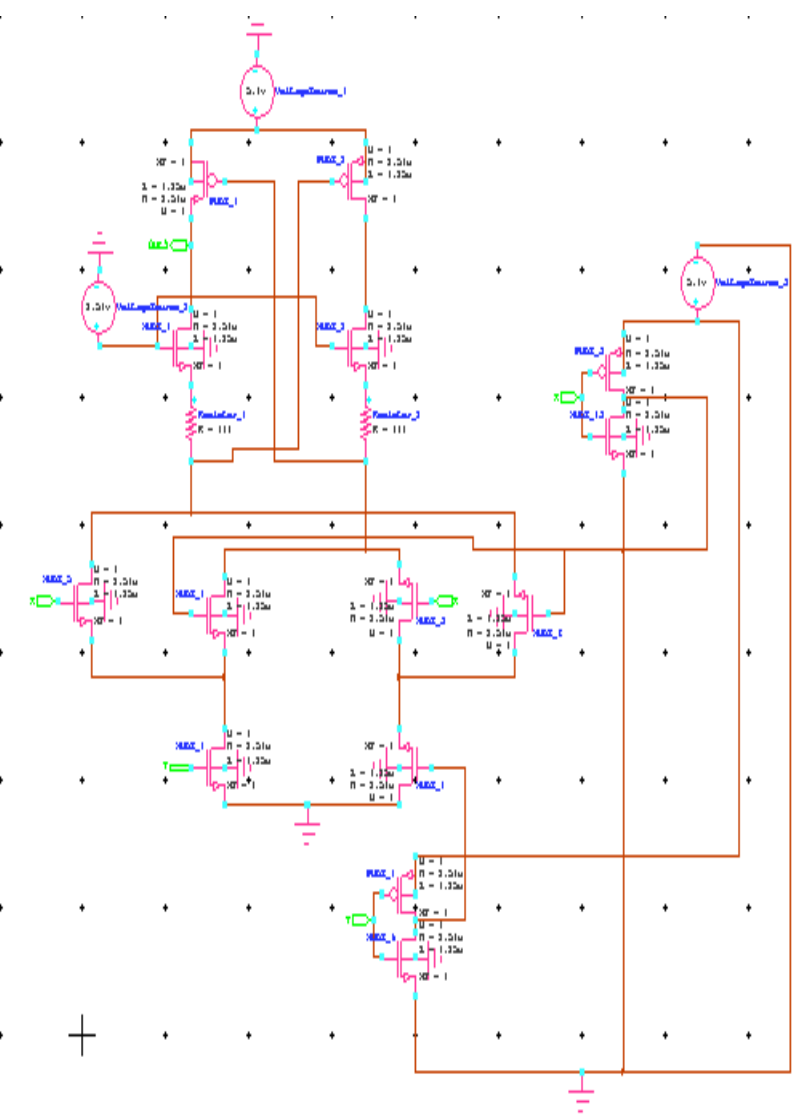

Fig 18: XOR circuit schematic

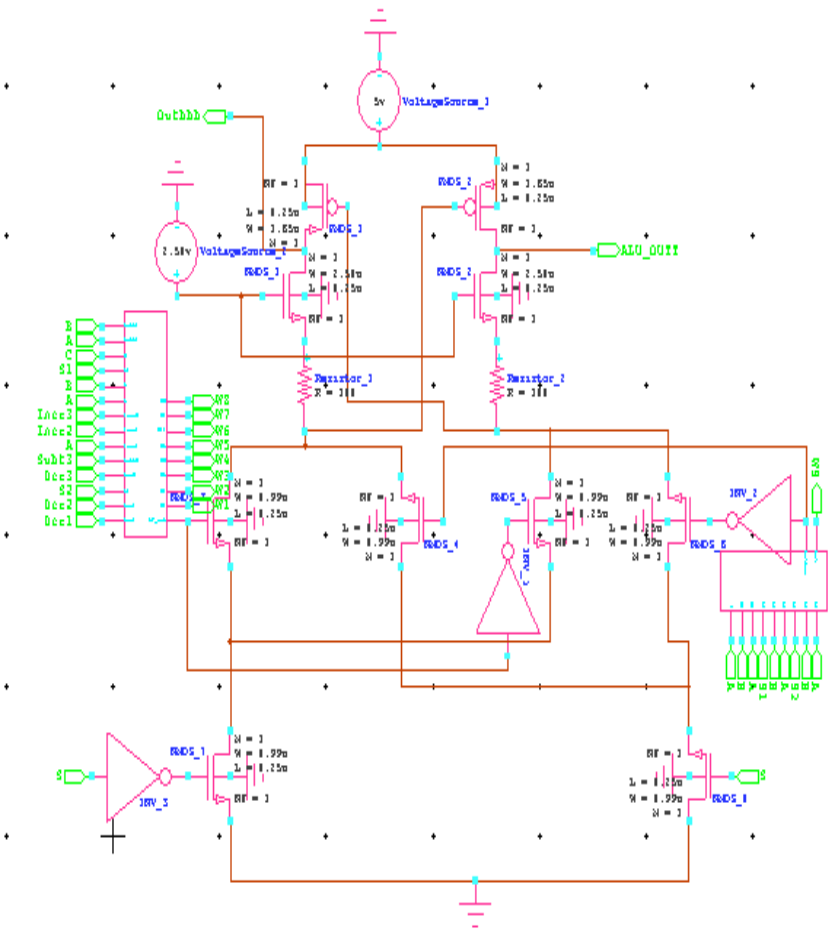

Fig 19: ALU circuit schematic

The output is taken across $2: 1$ mux [8]. The propagation delay, power dissipation and power delay product are calculated with power supply voltage as $5.0 \mathrm{v}$ and reference voltage as $2.58 \mathrm{~V}$ provided to reference NMOS transistors.

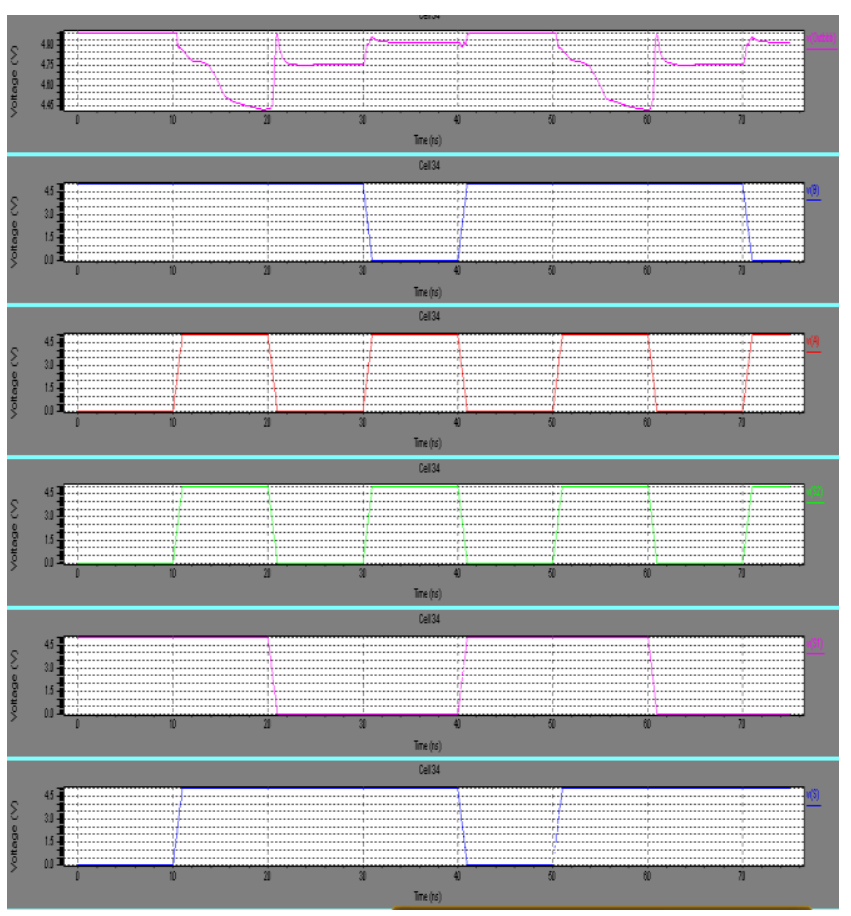

Fig 20: Simulation waveforms of ALU in W-edit

The given table shows the delay, power dissipation and power delay product calculated by using tanner eda v13.0 tool and technology file $130 \mathrm{~nm}$. 
Table 6. Performance parameters of ALU

\begin{tabular}{|c|c|c|c|}
\hline Function & Delay(ns) & Power(watt) & $\begin{array}{l}\text { PDP(watt } \\
\text { ns) }\end{array}$ \\
\hline AND & 2.02 & $\begin{array}{l}6.732498 \mathrm{e}- \\
007\end{array}$ & $\begin{array}{c}13.626575 \\
e-007\end{array}$ \\
\hline OR & 1.385 & $\begin{array}{l}3.561170 \mathrm{e}- \\
009\end{array}$ & $\begin{array}{c}4.93222045 \\
\text { e- } 009\end{array}$ \\
\hline XOR & 1.91 & $\begin{array}{l}9.706720 \mathrm{e}- \\
011\end{array}$ & $\begin{array}{c}18.5398352 \\
\mathrm{e}-011\end{array}$ \\
\hline INV & 1.2175 & $\begin{array}{c}9.217640 \mathrm{e}- \\
007\end{array}$ & $\begin{array}{c}11.2224767 \\
\mathrm{e}-007\end{array}$ \\
\hline Addition & 1.955 & $\begin{array}{c}2.218303 \mathrm{e}- \\
009\end{array}$ & $\begin{array}{c}3.58828129 \\
\text { e- } 009\end{array}$ \\
\hline Subtraction & 1.955 & $\begin{array}{c}1.835438 \mathrm{e}- \\
009\end{array}$ & $\begin{array}{c}3.58828129 \\
\text { e- } 009\end{array}$ \\
\hline Increment & 1.955 & $\begin{array}{c}1.537571 \mathrm{e}- \\
009\end{array}$ & $\begin{array}{c}3.005951305 \\
\text { e-009 }\end{array}$ \\
\hline Decrement & 1.955 & $\begin{array}{c}1.378934 \mathrm{e}- \\
009\end{array}$ & $\begin{array}{c}2.69581597 \\
\text { e-009 }\end{array}$ \\
\hline
\end{tabular}

\section{CONCLUSION}

The proposed full adder has better performance in terms of delay. It is observed with the simulation results that with adding resistor, propagation delay decreases in a fast way. But it is observed that on addition of capacitor in parallel to the resistor added in the circuit, the delay decreases gradually and the power dissipates more but less gradually in comparison to the modified full adder with added resistance only. Since priority is high speed, proposed full adder with only added resistor is used in designing ALU .After the analysis, the other components of ALU are designed with same modified DSL logic technique This proposed ALU can be used for high speed and low power applications.

\section{REFERENCES}

[1]A. P. Chandrakasan, S. Sheng and R. W. Brodersen, "Low Power CMOS Digital Design," IEEE Journal of Solidstate Circuits, vol. 27, No. 4, pp. 473-484, April 1999.
[2]Dimtrios Soudris,Christian Piguet,Costas Goustis, "Designing CMOS Circuits for Low Power (European Low-Power Initiative for Electronic System Design (Series))” ,Kluwer Academic Publishers,Oct 2002,pp.74$7 \mathrm{t} 8$

[3]P.Sheridan,C.M.Huizer,"AnExpression for the propagation delay of a Differential Split-Level (DSL) CMOS Logic Gate”,IEEE Journal of solid state circuits, Vol-22,No.3, June1987,pp.457-459

[4]Leo C.M.G. Pfennings,Etal.,"Differential Split Level CMOS Logic for Subnanosecond Speeds," IEEE Journal of Solid State Circuits, Vol. SC-20, No.5, Oct 1985,pp.1050-1055.

[5]Didem Z. Turker, Sunil P.Thakur, Edgar Sánchez-Sinencio "A DCVSL Delay Cell for Fast Low Power Frequency Synthesis Applications", IEEE Transactions on circuits and systems-I:Regular papers, June 2011, Vol. 58, No. 6,pp. 1225-1238

[6] S. M. Aziz, W. A. J. Waller, "On testability of Differential Split-Level CMOS Circuits", IEEE Proc.-Circuits Devices Syst., Vol. 141, No. 6, Dec 1994,pp.191-194

[7]L.G Heller, Etal.,"Cascode Voltage Switch Logic: A Differential CMOS Logic Family", Proceedings of 1984 IEEE International Solid-State Circuits Conference, pp. 16-17.

[8]Ila Gupta,Neha Arora,Dr.B.P.Singh,"Design and analysis of 2:1 multiplexer for high performance digital systems', IJECT, Vol. 3, Issue 1, Jan-March 2012

[9]Daniel D.Gajski, “Principles of Digital Design",Prentice Hall publishers,1997,pp.178-182

[10]Weiqiang Zhang, LinfengLi,Jianping Hu,"Design Techniques of P-Type CMOS Circuits for Gate-Leakage Reduction in Deep Sub-micron ICs",IEEE International Midwest Symposium ,Circuits and Systems, 2009. MWSCAS '09,2-5Aug, 2009, pp.551-554

[11]Dae Woon Kang,Yong-Bin Kim,"Design of enhanced differential cascode voltage switch logic(EDCVSL) circuits for high fan-in gate",ASIC/SOC conference, $2002,15^{\text {th }}$ annual IEEE international,2528sept 2002,pp.309-3013.

[12]Nehru K.,Shanmugam,A., Darmilathenmozhi,G.,"Design of low power ALU using 8T FA and PTL based MUX circuits",Advances in Engineering,Science and Management(ICAESM),IEEE international conference,30-31 march 2012,pp.724-730.

[13]M. Morris Mano,"Digital Logic design” ,Prentice Hall publishers, Aug 2001,pp.116-120 